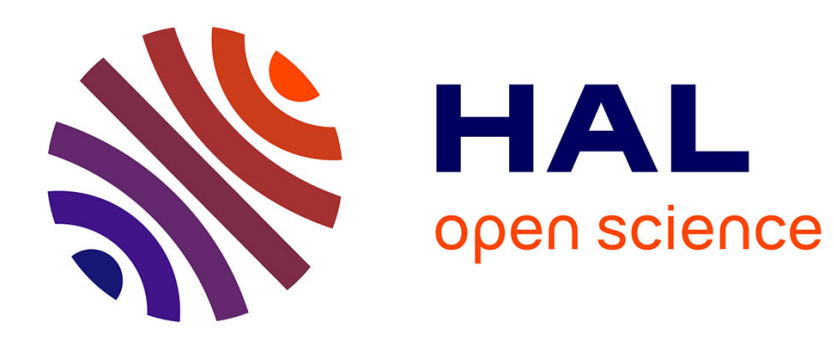

\title{
Étude de micelles ioniques par diffusion centrale des neutrons
}

\author{
C. Cabos, P. Delord
}

\section{To cite this version:}

C. Cabos, P. Delord. Étude de micelles ioniques par diffusion centrale des neutrons. Journal de Physique Lettres, 1977, 38 (17), pp.365-368. 10.1051/jphyslet:019770038017036500 . jpa-00231397

\section{HAL Id: jpa-00231397 https://hal.science/jpa-00231397}

Submitted on 1 Jan 1977

HAL is a multi-disciplinary open access archive for the deposit and dissemination of scientific research documents, whether they are published or not. The documents may come from teaching and research institutions in France or abroad, or from public or private research centers.
L'archive ouverte pluridisciplinaire HAL, est destinée au dépôt et à la diffusion de documents scientifiques de niveau recherche, publiés ou non, émanant des établissements d'enseignement et de recherche français ou étrangers, des laboratoires publics ou privés. 
Classification

Physics Abstracts

61.12

\title{
ÉTUDE DE MICELLES IONIQUES PAR DIFFUSION CENTRALE DES NEUTRONS
}

\author{
C. CABOS et P. DELORD
}

Groupe de dynamique des phases condensées $\left({ }^{*}\right)$ Laboratoire de Minéralogie et de Cristallographie Université des Sciences et Techniques du Languedoc, place Eugène-Bataillon, 34060 Montpellier, France

(Reçu le 18 juillet 1977, accepté le 29 juillet 1977)

\begin{abstract}
Résumé. - Nous avons étudié, par diffusion centrale des neutrons, des solutions micellaires d'un tensioactif ionique. Les diagrammes obtenus montrent la présence d'un anneau qu'on peut attribuer aux corrélations entre micelles. Une étude en contraste variable sur des solutions diluées nous a permis de déterminer les caractéristiques de la micelle.
\end{abstract}

Abstract. - We have studied by small angle scattering of neutrons, micellar solutions of ionic surfactant. The scattering diagram shows a correlation peak due to micelle micelle interaction. A contrast variation method using dilute solutions allows us to determine micellar characteristics.

1. Introduction. - Le problème de la structure des phases micellaires ioniques en solution aqueuse a déjà fait l'objet d'études par diffusion centrale des rayons X (D.C.R.X.) [1], [2], [3]. L'interprétation des diagrammes obtenus repose sur la connaissance de l'intensité diffusée à l'échelle absolue et sur son comportement asymptotique. Elle est rendue difficile par la superposition, aux effets de structure, des effets de corrélations entre micelles.

Plus récemment [4], cette analyse a été reprise en D.C.R.X. par la méthode du contraste variable appliquée à l'étude du complexe rhodopsine-détergent dans lequel l'amphiphile (DDAO) n'est pas ionique. Une étude analogue sur des solutions micellaires ioniques ne peut être envisagée aux rayons $X$ car toute variation du solvant, qui est le principe de cette méthode, ne peut être obtenue que par addition de substances organiques qui vont modifier certains paramètres micellaires (concentration micellaire critique, solvatation) ainsi que la structure micellaire, par le phénomène de solubilisation des additifs.

La diffusion des neutrons aux petits angles ne présente pas ces inconvénients. C'est, ici, la très grande différence entre les longueurs de diffusion des atomes $\mathrm{H}$ et $\mathrm{D}$ qui est à l'origine d'une importante variation de contraste dont la notion sera précisée dans le texte. Cette caractéristique est utilisée suivant deux techniques :

(*) Laboratoire associé au C.N.R.S.
- variation de la densité de longueur de diffusion $\mathrm{du}$ solvant par l'utilisation de mélanges connus $\mathrm{H}_{2} \mathrm{O}, \mathrm{D}_{2} \mathrm{O}$,

- variation de la densité de longueur de diffusion de la micelle à partir d'amphiphiles hydrogénés et deutérés.

Le montage expérimental de diffusion aux petits angles - D11 - installé sur le réacteur à haut flux de l'Institut Laue-Langevin de Grenoble, a permis cette étude dont nous présentons ici quelques résultats.

2. Méthode expérimentale. - 2.1 PRODUITS. L'amphiphile utilisé est le p. octylbenzènesulfonate de sodium. Le composé hydrogéné :

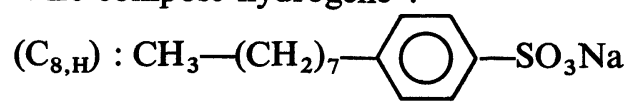

a été synthétisé au laboratoire suivant une technique déjà exposée [3]. Le même composé deutéré $\left(C_{8, D}\right)$ sur la chaîne paraffinique :

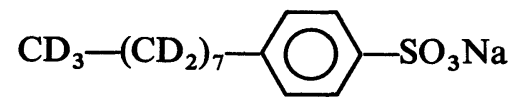

a été synthétisé au CEA de Saclay avec un taux de pureté de $99 \%$.

Des études préliminaires ont permis de déterminer la concentration micellaire critique (c.m.c.) de cet amphiphile : $1,2 \times 10^{-2}$ mole/1 à $30^{\circ} \mathrm{C}$ ainsi que la densité. Les solutions micellaires de $\mathrm{C}_{8, \mathrm{H}}$, de $\mathrm{C}_{8, \mathrm{D}}$ et des mélanges connus $\mathrm{C}_{8, \mathrm{H}}-\mathrm{C}_{8, \mathrm{p}}$ ont été étudiés dans des solvants variables $\mathrm{H}_{2} \mathrm{O}-\mathrm{D}_{2} \mathrm{O}$. Dans chaque cas, une série de solutions de concentrations décrois- 
santes ont été préparées, la concentration initiale étant de $0,05 \mathrm{~mole} / 1(c \approx 11,4 \mathrm{mg} / \mathrm{ml})$. Toutes les courbes de diffusion ont ainsi pu être extrapolées à la c.m.c.

2.2 MONTAGE EXPÉRIMENTAL. - La chambre D11 de diffusion des neutrons aux petits angles [5] permet de mesurer l'intensité diffusée $I(q)$ par un échantillon. $q=4 \pi \sin \theta / \lambda$ mesure le module du vecteur de l'espace réciproque où $2 \theta$ est l'angle de diffusion et $\lambda$ la longueur d'onde du rayonnement.

3. Etude théorique. - L'intensité diffusée par une solution diluée de particules identiques peut être explicitée si l'on connaît la fonction $\rho(\mathbf{r})$ qui décrit la variation de la densité de longueur de diffusion d'une particule. L'expression la plus complète de $\rho(\mathbf{r})$ [6] représente l'écart, par rapport au solvant, de la densité de longueur de diffusion dans la particule. Elle comporte deux termes :

$$
\rho(\mathbf{r})=\bar{\rho} \rho_{\mathrm{c}}(\mathbf{r})+\rho_{\mathrm{s}}(\mathbf{r})
$$

avec $\bar{\rho}=\langle\rho\rangle-\rho_{0}$ où $\langle\rho\rangle$ et $\rho_{0}$ sont respectivement les densité moyennes de longueur de diffusion de la particule et du solvant.

$\rho_{\mathrm{c}}(\mathbf{r})$ décrit la forme d'une particule pouvant être partiellement accessible au solvant. $\rho_{\mathrm{c}}(\mathbf{r})=1$ dans la partie hydrophobe (non solvatée) de la micelle. $0<\rho_{\mathrm{c}}(\mathbf{r}) \leqslant 1$ dans la partie polaire solvatée.

$\rho_{\mathrm{s}}(\mathbf{r})$ représente les fluctuations de la densité de longueur de diffusion dans la particule autour de $\langle\rho\rangle$.

Le volume $V_{\mathrm{c}}$ d'une particule, exclue au solvant, est ainsi défini par :

$$
V_{\mathrm{c}}=\int \rho_{\mathrm{c}}(\mathbf{r}) \mathrm{d}^{3} r
$$

3.1 Diffusion A L'ANGLe Nul. - L'intensité diffusée aux petits angles s'exprime comme la somme de trois fonctions [6].

$$
I(q)=\bar{\rho}^{2} I_{\mathrm{c}}(q)+\bar{\rho} I_{\mathrm{cs}}(q)+I_{\mathrm{s}}(q)
$$

où $I_{\mathrm{c}}(q)$ et $I_{\mathrm{s}}(q)$ sont les fonctions de diffusion provenant de $\rho_{\mathrm{c}}$ et $\rho_{\mathrm{s}}$ et $I_{\mathrm{cs}}$ traduit les corrélations entre $I_{\mathrm{c}}$ et $I_{\mathrm{s}}$.

$I_{\mathrm{cs}}(q)$ et $I_{\mathrm{s}}(q)$ tendent vers zéro pour $q \rightarrow 0$.

Pour une particule isolée l'intensité diffusée à l'angle nul vérifie la relation :

$$
\left[i_{1}\left(0, \rho_{0}\right)\right]^{1 / 2}=\left(\langle\rho\rangle-\rho_{0}\right) V_{\mathrm{c}} .
$$

3.2 RAYON DE GIRATION. - Le rayon de giration d'une particule est définie par la relation :

$$
R^{2}=\frac{\int \rho(\mathbf{r}) r^{2} \mathrm{~d}^{3} r}{\int \rho(\mathbf{r}) \mathrm{d}^{3} r} .
$$

$R$ dépend donc de $\rho_{0}$ suivant une loi dont l'expression générale [7] se simplifie dans le cas d'une particule centrosymétrique et qui s'écrit :

$$
R^{2}=R_{\mathrm{c}}^{2}+\frac{\alpha}{\langle\rho\rangle-\rho_{0}}
$$

avec

$$
\alpha=\frac{m_{2, \rho}}{V_{\mathrm{c}}} \quad \text { et } \quad m_{2, \rho}=\int \rho_{\mathrm{s}}(r) r^{2} \mathrm{~d}^{3} r
$$

et $R_{\mathrm{c}}$ rayon de giration de $\rho_{\mathrm{c}}(r)$, indépendant de $\bar{\rho}$.

4. Résultats. - L'existence de corrélations entre micelles ioniques se manifeste par la présence d'un maximum dont la position donne, en première approximation, la distance intermicellaire qui correspond bien à la distance moyenne déduite de la concentration en matière micellaire. Cet effet ne se manifeste pas sur des micelles non ioniques et paraît caractéristique d'interactions électrostatiques. Ceci nous a conduit à utiliser des solutions très diluées pour minimiser ces interactions et atteindre l'information particulaire.

Il est à noter que les effets des corrélations ne disparaissent pas, mais ne perturbent pas de manière notable la loi de Guinier.

4.1 DiffuSION A L'ANGLE NUL. - La racine carrée de l'intensité diffusée à l'angle nul est d'après (1),

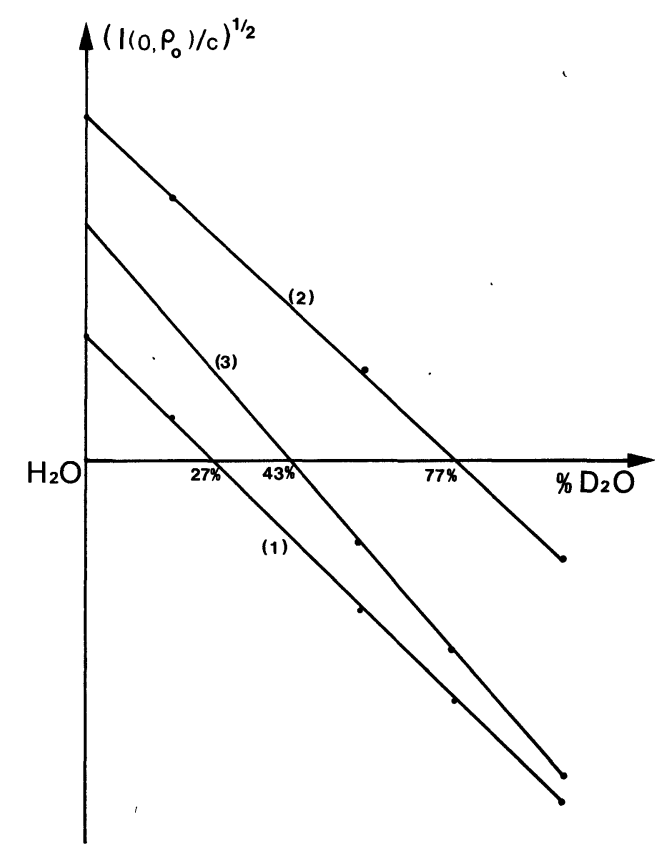

Fig. 1. - La racine carrée de $I\left(0, \rho_{0}\right) / c$ (à une échelle arbitraire) est une fonction linéaire de la densité de longueur de diffusion du solvant
(1) $\mathrm{C}_{8 \mathrm{H}}$
(2) $\mathrm{C}_{8 \mathrm{H}}$
(3) Hémoglobine.

[The square root of $I\left(0, \rho_{0}\right) / c$ (in arbitrary unity) is a linear function of the scattering length density of the solvant.

(1) $\mathrm{C}_{8 \mathrm{H}}$

(2) $\mathrm{C}_{8 \mathrm{D}}$

(3) Hemoglobine.] 
une fonction linéaire de la densité de longueur de diffusion du solvant. Ceci reste valable dans le cas de mesures relatives de l'intensité. La figure 1 représente $\left[I\left(0, \rho_{0}\right) / c\right]^{1 / 2}$ pour $\mathrm{C}_{8, \mathrm{H}}$ et $\mathrm{C}_{8, \mathrm{D}}$.

L'intersection des deux droites représentées avec l'axe des abscisses permet de calculer $\langle\rho\rangle$ dans chaque cas.

Ainsi :

$$
\begin{aligned}
& \langle\rho\rangle \mathrm{C}_{8, \mathrm{H}}=1,25 \times 10^{10} \mathrm{~cm}^{-2} \\
& \langle\rho\rangle \mathrm{C}_{8, \mathrm{D}}=4,7 \times 10^{10} \mathrm{~cm}^{-2}
\end{aligned}
$$

Des mélanges réalisés à partir de $C_{8, H}$ et $C_{8, D}$ ont permis d'étudier la variation de $\left[I\left(0, \rho_{0}\right) / c\right]^{1 / 2}$ pour plusieurs valeurs de la densité de longueur de diffusion de la partie paraffinique de la micelle $: \rho_{\text {par }}$.

Cette densité peut être calculée dans l'hypothèse classique d'une structure désordonnée, quasi liquide, de cette région interne de la micelle.

De la mesure de $\langle\rho\rangle$ et du calcul de $\rho_{\text {par }}$ on peut ainsi déduire :

$x$, fraction de volume occupé par les parties paraffiniques

$$
x=\frac{V_{\text {par }}}{V_{\mathrm{c}}} \text { où } \quad V_{\mathrm{par}} \text { est le volume paraffinique . }
$$

$\rho_{\text {pol }}$, densité de longueur de diffusion de la partie polaire de la micelle non solvatée.

Par la relation $:\langle\rho\rangle=x \rho_{\mathrm{par}}+(1-x) \rho_{\mathrm{pol}}$ on obtient : $x=0,55 \pm 0,02$

$$
\rho_{\text {pol }}=(3,2 \pm 0,1) \times 10^{10} \mathrm{~cm}^{-2}
$$

Une exploitation plus complète de la formule (1) nécessite la connaissance de l'intensité à l'échelle absolue. Ceci a pu être obtenu en normant les intensités expérimentales par la diffusion, réalisée dans les mêmes conditions, d'une protéine bien connue par d'autres méthodes : l'hémoglobine.

$\left[I\left(0, \rho_{0}\right) / c\right]^{1 / 2}$ dans le cas de l'hémoglobine est représenté sur la figure 1 . $\left\langle\rho_{\mathrm{h}}\right\rangle$ est obtenu comme précédemment. Sa valeur correspond à la densité de longueur de diffusion du solvant à $43 \% \mathrm{D}_{2} \mathrm{O}$, c'est-à-dire $\left\langle\rho_{\mathrm{h}}\right\rangle=2,4 \times 10^{10} \mathrm{~cm}^{-2}$. La longueur de diffusion totale $\Sigma_{b}$ de la protéine peut être calculée à partir de sa composition chimique et le volume $V_{\mathrm{h}}$ de la particule s'exprime suivant :

$$
V_{\mathrm{h}}=\frac{\Sigma_{b}}{\langle\rho\rangle}=80400 \AA^{3} .
$$

En divisant $V_{\mathrm{h}}$ par la masse moléculaire, on obtient le volume spécifique partiel

$$
\bar{v}=V_{\mathrm{h}} \frac{N}{M_{\mathrm{h}}}=0,749 \mathrm{~cm}^{3} / \mathrm{g}
$$

qui est la valeur généralement adoptée.

Connaissant $V_{\mathrm{h}}, M_{\mathrm{h}}$ et $\bar{v}$ le rapport des pentes des droites représentées sur la figure 1 permet de déterminer la masse molaire et le volume de la micelle $M_{\mathrm{C}_{8}} \approx 23300, V_{\mathrm{C}} \approx 37800 \AA^{3}$. Notons que la précision des mesures ne permet pas de différencier
$M_{\mathrm{C}_{8 \mathrm{H}}}$ et $M_{\mathrm{C}_{8 \mathrm{D}}}$ dont les masses molaires diffèrent théoriquement de $5 \%$. Le nombre $n$ de monomères par micelle peut être calculé si l'on connaît le degré d'association des contre-ions. Ce nombre, qui représente le rapport entre le nombre d'ions $\mathrm{Na}^{+}$liés à la micelle au nombre de monomères par micelle est voisin de 0,6 [8] et conduit à $n=82 \pm 3$.

Il est maintenant possible de tracer à l'échelle absolue $\left(\left[i_{1}\left(0, \rho_{0}\right)\right]^{1 / 2}\right)$ pour une micelle et pour chaque valeur de $\rho_{\text {par }}$ correspondant aux différents mélanges $\mathrm{C}_{8 \mathrm{H}}, \mathrm{C}_{8 \mathrm{D}}$. De ce réseau de droites parallèles, dont la pente est égale à $V_{\mathrm{C}}$, on peut déduire $\left(\left[i_{1}\left(0, \rho_{\mathrm{par}}\right)\right]^{1 / 2}\right)$ pour un solvant donné.

La figure 2 représente le graphe de cette fonction pour un solvant à $27 \% \mathrm{D}_{2} \mathrm{O}$. L'équation de la droite représentée se déduit de la formule (1) :

$\left[i_{1}\left(0, \rho_{\mathrm{par}}\right)\right]^{1 / 2}=\rho_{\mathrm{par}} V_{\mathrm{par}}+\left[(1-x) \rho_{\mathrm{pol}}-\rho_{0}\right] V_{\mathrm{C}}$.

La mesure de la pente est $V_{\text {par }}$. On obtient $V_{\text {par }}=21000 \AA^{3}$ valeur qui donne $x=0,55$.

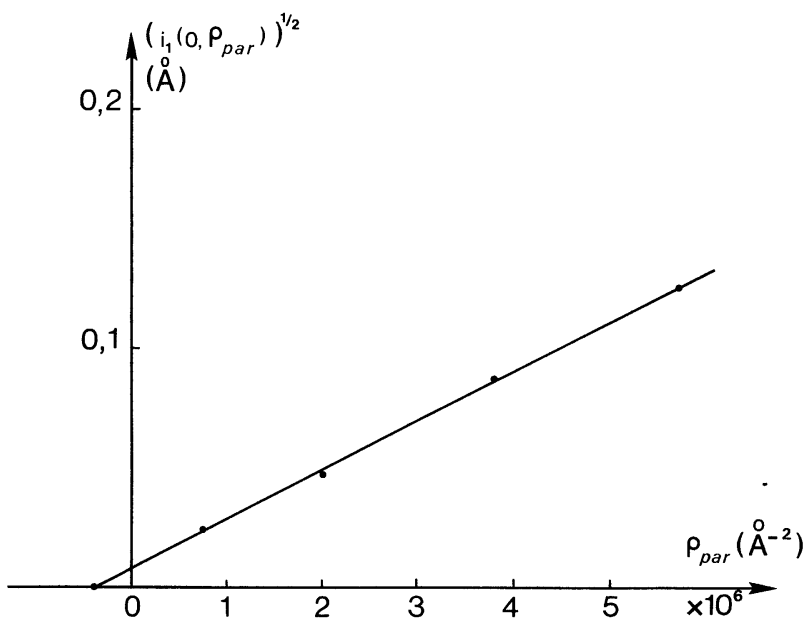

Fig. 2. - Pour un solvant donné $\left(27 \% \mathrm{D}_{2} \mathrm{O}\right)$ la racine carrée de $i_{1}(0)$ est une fonction linéaire de la densité de longueur de diffusion de la partie paraffinique de la micelle.

[For a given solvent $\left(27 \% \mathrm{D}_{2} \mathrm{O}\right)$ the square root of $i_{1}(0)$ is a linear function of the scattering length density of the paraffinic part of the micelle.]

4.2 RAYON DE GIRATION. - Le rayon de giration obtenu expérimentalement varie en fonction du contraste moyen suivant la loi linéaire (2) qui est illustré - dans le cas de solutions micellaires de $\mathrm{C}_{8 \mathrm{H}}$ - sur la figure 3. La droite représentée permet de calculer $\alpha$ et $R_{\mathrm{c}}^{2}$

$$
\begin{aligned}
\alpha & =16 \times 10^{-5} \\
R_{\mathrm{c}}^{2} & =240 \AA^{2} .
\end{aligned}
$$

Connaissant $V_{\mathrm{c}}$ on peut calculer le moment d'ordre 2 des fluctuations de la densité de longueur de diffusion à l'intérieur de la micelle.

$$
m_{2, \rho}=11 \times 10^{-5} \times 37800=4,16 \AA^{3} .
$$

On pourra suivre l'évolution de $m_{2, \rho}\left(\rho_{\text {par }}\right)$ à partir des mélanges $\mathrm{C}_{8 \mathrm{H}}, \mathrm{C}_{8 \mathrm{D}}$. La formule (2) permet par 


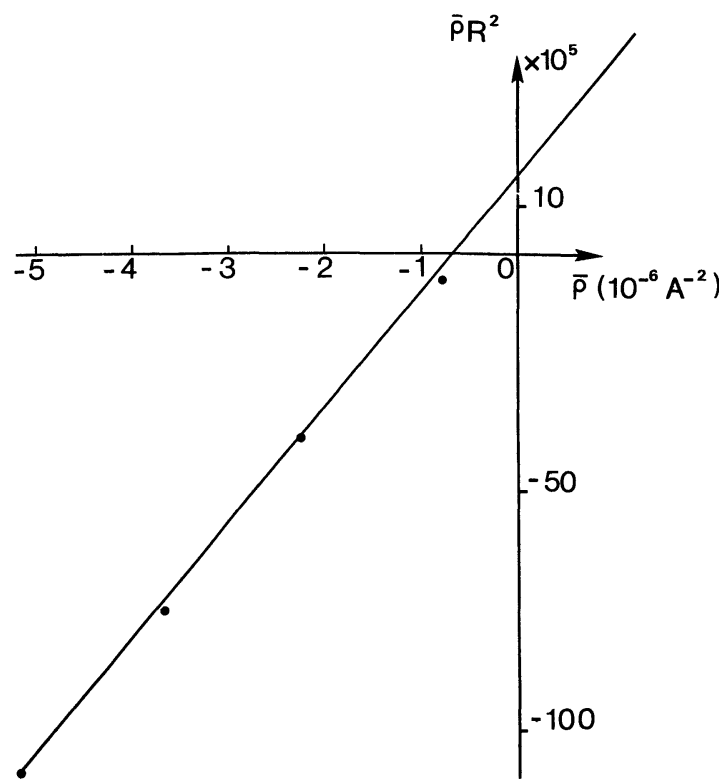

FIG. 3. - Le carré du rayon de giration est une fonction linéaire de l'inverse du contraste.

[The square of the radius of gyration is a linear function of the reciprocal of the contrast.]

ailleurs de calculer les rayons de giration $R_{\text {par }}$ et $R_{\mathrm{pol}}$ de la partie paraffinique et de la micelle solvatée. En effet, si l'on donne à $\rho_{0}$ la valeur $\rho_{\text {par }}$, la particule $v u e$ est celle dont le rayon de giration est $R_{\mathrm{pol}}$. Inversement, pour $\rho_{0}=\rho_{\text {pol }}$ tout se passe comme si la particule de densité homogène $\langle\rho\rangle$ avait un rayon de giration $R_{\mathrm{pol}}$.

Le calcul conduit à :

$$
\begin{aligned}
& R_{\mathrm{par}}=12,6 \AA \\
& R_{\mathrm{pol}}=20,5 \AA .
\end{aligned}
$$

Dans l'hypothèse d'une solution monodisperse de particules sphériques, on peut calculer le volume occupé par la partie paraffinique et le volume total de la micelle solvatée

$$
\begin{aligned}
V_{\mathrm{par}} & =18000 \AA^{3} . \\
V & =77000 \AA^{3} .
\end{aligned}
$$

L'analyse complète des résultats obtenus à partir de $R^{2}\left(\rho_{0}\right)$ pour les différentes valeurs de $\rho_{\text {par }}$ permettra de préciser ces valeurs numériques. Notons que cette analyse, indépendante de 4.1 conduit néanmoins à des résultats très voisins. Elle permet par ailleurs de connaître approximativement le volume de solvant qui baigne la micelle $\left(V-V_{c}\right)$ et celui qui entoure la partie polaire de chaque monomère.

5. Conclusion. - La diffusion neutronique présente de gros avantages sur la diffusion des rayons $\mathrm{X}$. A une très grande souplesse sur le choix de la longueur d'onde, elle associe, dans le cas de milieux hydrocarbonés, en solution aqueuse, une gamme très lạrge de contrastes possibles.

Dans le cas de milieux tensioactifs ioniques, cela se traduit par des contrastes remarquables qui permettent de travailler à des concentrations qu'on ne pourrait étudier par rayons $\mathrm{X}$ et par des méthodes de contraste variable basées sur la différence de longueur de diffusion entre $H$ et $D$ qui ne perturbent pas le milieu.

A la suite de cette étude, le milieu micellaire ionique apparaît comme un milieu où les interactions électrostatiques jouent un rôle prépondérant même aux concentrations faibles. Le fait que la taille de la micelle reste petite par rapport aux distances intermicellaires, nous a permis d'obtenir les caractéristiques de la micelle sans autre hypothèse que celle d'une structure quasi liquide des paraffines.

Cette étude n'est qu'une première approche qui admet une variation linéaire de l'intensité à l'angle nul en fonction du contraste : ce qui n'est qu'une première approximation pour un milieu polydispersé. Nous pensons prochainement examiner les effets de la polydispersité qui se manifestent aux contrastes faibles. Cette étude, qui pourra aussi permettre des études structurales plus fines, peut être d'un grand intérêt pour étudier les effets de la force ionique sur la dispersité des milieux micellaires ioniques. Nous pensons aussi étudier, à force ionique constante, l'effet de la taille des contre-ions sur la dimension des micelles qui peuvent d'une part introduire une courbure spontanée du milieu, d'autre part modifier les interactions électrostatiques [9].

Remerciements. - Nous tenons à remercier particulièrement MM. Volino F. et Dianoux J. de l'I.L.L. de Grenoble pour leur collaboration sur le plan expérimental, ainsi que MM. Chabre $M$. et Osborne B. H. du C.E.N.G. de l'aide qu'ils nous ont apportée pour l'étude de l'hémoglobine en contraste variable.

\section{Bibliographie}

[1] Reiss-Husson, F., Thèse, Strasbourg (1963).

[2] Svens, B. et Rosenholm, B., J. Colloid Interface Sci. 44 (1973) 495.

[3] Cabos, C., Delord, P. et Rouvière, J., J. Appl. Cryst. 10 (1977) 37.

[4] Sardet, C., Tardieu, A. et Luzzati, V., J. Mol. Biol. 105 (1976) 383.
[5] Schmatz, W., Springer, T. et Schelten, J., J. Appl. Cryst. 7 (1974) 96

[6] Stuhrmann, H. B., J. Appl. Cryst. 7 (1974) 173.

[7] Luzzati, V., Tardieu, A., Mateu, L. et Stuhrmann, H. B., J. Mol. Biol. 101 (1976) 115.

[8] Kamenka, N., Lindman, B., Fontell, K., Chorro, M. et Brun, B., C.R. Hebd. Séan. Acad. Sci. C. 284 (1977) 403.

[9] Parodi, O., J. Physique Lett. 37 (1976) L-295.

Commission paritaire № 59.024 